\title{
Des races localement adaptées et adoptées, une condition de la durabilité des activités d'élevage
}

\author{
Nathalie Couix ${ }^{1, *}$, Claire Gaillard ${ }^{2,3}$, Anne Lauvie ${ }^{4}$, Sylvie Mugnier ${ }^{2,3}$ et Étienne Verrier ${ }^{5}$ \\ 1 AGIR, Université de Toulouse, INRA, INPT, INP-EI PURPAN, 31326 Castanet Tolosan, France \\ 2 UMR Métafort, AgroSup Dijon, BP 87999, 21079 Dijon, France \\ ${ }^{3}$ INRA n ${ }^{\circ} 1273,63000$ Clermont-Ferrand, France \\ ${ }^{4}$ UMR 0868 Systèmes d'élevage méditerranéens et tropicaux, INRA-CIRAD-Montpellier SupAgro, 2, place Viala, 34060 Montpellier \\ cedex 01, France \\ ${ }^{5}$ GABI, INRA, AgroParisTech, Université Paris-Saclay, 75005 Paris, France
}

\begin{abstract}
Résumé - Dans une perspective de durabilité des activités d'élevage, le recours à la diversité des ressources génétiques animales disponibles est présenté comme un levier potentiel. Les races locales notamment sont mises en avant pour leur adaptation aux territoires et aux systèmes d'élevage de leur berceau d'origine. Cet article vise à comprendre comment la mobilisation de ressources animales, locales ou non, peut contribuer à une adaptation des systèmes de production et à leur durabilité. Deux études de cas ont été conduites sur l'élevage bovin laitier dans le Grand Ouest de la France, l'une concernant deux races récemment introduites dans la région, la Montbéliarde et la Simmental, l'autre concernant la Bretonne Pie Noir, race locale à petit effectif. Les résultats de ces études ont été mis en perspective à l'aide d'un cadre conceptuel inspiré de la théorie pragmatiste de l'enquête. Cela nous conduit à réinterroger la notion même de " race localement adaptée » et à lui associer celle de race « localement adoptée ».
\end{abstract}

Mots clés : génétique animale / race locale / adaptation / durabilité / système d'élevage

\begin{abstract}
Breeds both locally adapted and locally adopted, a condition for the sustainability of livestock activities. Using the diversity of animal genetic resources is considered as one possible way toward sustainability of livestock farming systems. Emphasis is generally put on local breeds regarding their adaptation to the conditions and farming systems in their territory of origin. In this paper, we show how using some breeds, local or not, may modify farming systems and, then, contribute to their sustainability. Two case studies were conducted on dairy farming systems in Western France, one dealing with two cattle breeds recently introduced in this region, namely the Montbéliarde and Simmental breeds, the other one dealing with the rare and local Bretonne Pie Noir breed. The results of these studies were interpreted within a conceptual framework inspired by the pragmatist theory of inquiry. We conclude that the concept of "locally adapted breed" has to be associated with the concept of "locally adopted breed".
\end{abstract}

Keywords: animal genetic resource / local breed / adaptation / sustainability / animal farming system

\section{Introduction}

Dans une perspective de durabilité des activités d'élevage, le recours à la diversité des ressources génétiques animales disponibles est présenté comme un levier, cette diversité étant vue comme un facteur de résilience des systèmes (Dumont et al., 2013). Parmi ces ressources, les races locales sont souvent mises en avant pour leur adaptation aux territoires et aux systèmes d'élevage de leur berceau d'origine. En

\footnotetext{
$\bar{*}$ Auteur de correspondance : nathalie.couix@inra.fr
}

témoignent les sites web des organismes de sélection de ces races, qui mettent en avant cette adaptation (par exemple, voir les sites des races bovines Bretonne Pie Noir, Vosgienne ou Villard de Lans). D'autres organismes de gestion de races locales font état d'une adaptation des races à une diversité de systèmes, au-delà de l'adaptation à un territoire d'élevage berceau, du fait de leurs aptitudes (voir par exemple le site de la race bovine Salers). Dans tous les cas, les aptitudes spécifiques explicitées correspondent à celles attendues pour des systèmes d'élevage plus économes et autonomes, répondant à des enjeux de durabilité : valorisation de fourrages grossiers, élevage de plein air, recours à la marche, qualité des produits, etc. La 
question se pose donc de savoir comment, le cas échéant, les éleveurs choisissent la race qu'ils mobilisent avec un objectif de durabilité économique, sociale et/ou environnementale de leurs activités d'élevage, sur un territoire d'élevage donné.

En termes d'adaptation, il y a en fait une co-évolution des pratiques d'élevage et des aptitudes de la race afin de répondre aux objectifs des éleveurs. La mécanisation de la traite en est un bon exemple. Pour faciliter le travail des éleveurs et augmenter la production laitière, la traite mécanisée a été développée. Les machines à traire ont été adaptées à la morphologie des trayons mais la vache aussi a été sélectionnée pour être traite de façon mécanique (Labussière et Richard, 1965 ; Verrier et al., 2010). D'une manière générale, le système de production trouve son équilibre lorsque la conduite du troupeau est en adéquation avec les aptitudes de la race (Vissac, 1993). Par exemple, la race bovine Holstein a été adaptée à des systèmes intensifs où l'alimentation est calée sur les besoins de la vache en vue d'une forte productivité. À l'opposé, d'autres races bovines, mixtes ou rustiques, moins exigeantes en intrants, sont ou ont été adaptées à des systèmes qui s'affranchissent moins des conditions de milieu, comme des systèmes herbagers ou pastoraux. Les femelles de ces races mixtes ou rustiques ont une meilleure aptitude à la reproduction mais une plus faible productivité (Piccand et al., 2011, pour le cas de la production laitière).

La race très spécialisée peut néanmoins être mobilisée dans différents systèmes de production. Ainsi la race Holstein peut être utilisée dans des systèmes à bas intrants essentiellement herbagers comme c'est le cas en Nouvelle-Zélande, entraînant une conduite du troupeau plus compliquée, contrairement à l'utilisation des races Montbéliarde et Normande (Coquil et al., 2009 ; Delaby et Fiorelli, 2014). En Nouvelle-Zélande, l'utilisation de la race Holstein dans ces systèmes herbagers passe par une mobilisation de taureaux Holstein génétiquement « originaux » sur le plan de leur pedigree (Mattalia et al., 2006). Toutefois, les taureaux d'insémination néozélandais disponibles sur le marché mondial sont très peu nombreux et ils ressortent très mal classés dans les résultats d'évaluation génétique internationale, pour l'objectif global de sélection de même que pour le caractère « reproduction » (http://primhols tein.com/index/index-taureaux-interbull/ consulté le 17/11/ 2016). On comprend alors que l'existence de rares taureaux d'insémination néozélandais n'ait pas été perçue comme une solution aux problèmes de fertilité rencontrés en race Holstein en France.

Dans une perspective de durabilité des activités d'élevage et de transition agro-écologique, la caractérisation de l'adaptation des races et des compromis entre adaptation et production dans la gestion de ces races constitue un enjeu de recherche clé (Dumont et al., 2014). A partir de deux études de cas, cet article vise à comprendre comment des éleveurs font le choix des ressources animales qu'ils mobilisent pour répondre à des objectifs de durabilité des systèmes d'élevage. Il vise en outre à préciser le lien entre le caractère local des races et les modalités de cette réponse.

\section{Matériel et méthode}

\subsection{Deux études de cas}

Deux études de cas ont été conduites, indépendamment l'une de l'autre, sur l'élevage bovin laitier dans le Grand Ouest de la France. Cette grande région est emblématique d'une production intensive et son cheptel de vaches laitières est composé à $75 \%$ de la race Holstein, c'est-à-dire la race la plus productive. Des éleveurs de plus en plus nombreux, même s'ils restent minoritaires, interrogent ici le compromis entre adaptation de la race et système de production, et cherchent à développer des systèmes alternatifs par opposition au système dominant. Une première étude de cas s'intéresse à des éleveurs qui ont fait le choix de s'installer avec la race Bretonne Pie Noir (BPN), race qui, bien que locale, a failli disparaître au détriment de la Normande puis de la Holstein. La seconde étude de cas s'intéresse à des éleveurs, initialement installés avec des Holstein, qui ont transformé leur système de production en mobilisant deux races bovines originaires de l'Est de la France : la Montbéliarde depuis la fin des années 70 et la Simmental Française depuis la fin des années 80 .

Dans le premier cas, 32 entretiens compréhensifs (Kaufmann, 1996) ont été conduits en 2012 auprès d'éleveurs installés avec des vaches BPN, 19 auprès d'éleveurs professionnels et 13 auprès d'amateurs ou retraités. Le guide d'entretien couvrait des thèmes tels que la trajectoire des éleveurs, les motifs du choix de la race, le système de production, la gestion de la reproduction, etc. Les éleveurs ont été choisis afin de couvrir une diversité de situations selon quatre critères : (i) localisation dans les départements bretons (Morbihan, Finistère, Côtes d'Armor, Ille et Vilaine) ainsi qu'en Loire Atlantique, (ii) caractère professionnel ou amateur (selon les listes des adhérents à l'Union Bretonne Pie Noir), (iii) laitier ou allaitant et enfin (iv) ancienneté de l'installation. Il s'agissait de s'assurer que toutes les modalités étaient représentées dans notre échantillon et non d'avoir un échantillon statistiquement représentatif des différentes catégories dans la population totale d'éleveurs de BPN. Les entretiens ont été retranscrits et ont donné lieu à une analyse thématique avec le logiciel N'Vivo.

Dans le second cas, 40 entretiens semi-directifs ont été conduits en 2011 auprès d'éleveurs ayant partiellement ou totalement remplacé les vaches Holstein de leur troupeau par des vaches Montbéliardes (20 éleveurs) ou Simmental Françaises (20 éleveurs), dans cinq départements du Grand Ouest (Ille-et-Vilaine, Loire-Atlantique, Maine-et-Loire, Mayenne et Morbihan). Un échantillon stratifié a été constitué à partir de la proportion de vaches de race Montbéliarde ou Simmental dans le troupeau (avec un seuil minimal de $25 \%$ ) parmi 552 exploitations pour la race Montbéliarde et 30 pour la race Simmental. Ces entretiens visaient à comprendre les motivations des éleveurs ayant opéré ces changements. Les entretiens ont porté sur la trajectoire des éleveurs, les raisons de l'introduction d'une nouvelle race dans le troupeau, ses modalités de conduite ainsi que ses avantages et ses inconvénients tels que perçus par les éleveurs. Une typologie réalisée à l'aide d'une analyse factorielle multiple des correspondances a permis de mettre en évidence différentes motivations des éleveurs et d'analyser leur lien avec la transformation des systèmes de production.

\subsection{Une mise en perspective des cas au travers d'un cadre conceptuel inspiré de la théorie de l'enquête de J. Dewey}

Le cadre conceptuel mobilisé propose d'interpréter les transformations et adaptations des systèmes d'élevage comme 
des processus «d'enquête» au sens de Dewey (1938). L'enquête peut en effet être considérée comme une des figures de l'adaptation (Zask, 2008). Dans une situation qu'il ressent en déséquilibre, l'éleveur va conduire une enquête pour tenter de définir le problème à résoudre, rechercher dans la situation les moyens qui se prêtent le mieux à l'action et au rétablissement d'une situation plus «déterminée », plus cohérente et moins incertaine, dans laquelle il va pouvoir poursuivre ses activités. Dans cette approche pragmatiste, la situation est émergente, elle se construit dans les interactions réciproques - les transactions - entre l'individu (singulier ou pluriel) et son environnement. Les savoirs sont élaborés dans l'expérience que l'individu fait du monde. L'enquête est déclenchée lorsque des éléments de la situation ne font plus sens, que les interactions entre l'individu et son environnement deviennent plus compliquées. Elle cherche alors à reconstruire une situation plus favorable à la poursuite des activités de l'individu. Ce faisant, la situation se transforme en même temps que l'individu. Aussi, à la différence du contexte qui est pensé indépendant de l'individu et auquel on s'ajuste sans avoir de réelles prises sur lui, la situation se construit en continu au fil de l'enquête.

Si le contexte des élevages peut être le même, les situations sont ainsi propres à chaque éleveur, à son expérience et aux savoirs élaborés au cours de cette expérience. Les problèmes identifiés par les éleveurs ne sont pas identiques. Les solutions ne s'imposent pas, elles sont construites au cours de l'enquête qui contribue elle-même à redéfinir la situation. Les ressources mobilisées au fil de ce processus peuvent être de tous ordres : matérielles, informationnelles, cognitives, organisationnelles. Il peut s'agir de mobiliser une nouvelle race, de mobiliser des subventions auprès d'organismes divers, de faire appel à des savoir-faire élaborés au cours d'expériences précédentes ou partagés dans un collectif d'éleveurs, de suivre une formation, de mobiliser des voisins pour une action collective autour de questions partagées, etc.

Dans la suite, nous allons interpréter les deux cas au travers de ce cadre, afin de comprendre les processus d'enquête qui ont conduit à la mobilisation, à des fins de durabilité des activités d'élevage, de la Bretonne Pie Noir dans un cas et de la Montbéliarde ou de la Simmental dans l'autre.

\section{Résultats}

\subsection{La BPN localement ré-adoptée}

Dans le cadre du plan de sauvegarde de la race BPN en 1976, 46 éleveurs de BPN ont signé un contrat de sauvegarde. Aujourd'hui, on en compte environ 340, essentiellement dans le Grand Ouest. Cette évolution substantielle du nombre d'éleveurs de BPN nous conduit à parler d'une ré-adoption de la race, même si elle reste très minoritaire.

Les entretiens réalisés mettent en évidence que les éleveurs rencontrés ont choisi la race BPN, au moment de leur installation, au cours d'un processus d'enquête qui visait la construction d'une situation cohérente avec leur projet de vie et leur conception de leur environnement et de l'agriculture. Chaque éleveur a utilisé alors les ressources qui lui paraissaient mobilisables dans sa situation, dont la race bovine locale. Chez ces éleveurs, la dimension locale fait sens dans le processus d'enquête et mérite d'être explicitée comme un construit plutôt que comme une propriété ontologique des ressources mobilisées. Le caractère local de la race est en effet un argument de choix pour plusieurs éleveurs qui lui donnent cependant des sens différents. Il peut renvoyer aux aptitudes fonctionnelles et au comportement des animaux, à l'adaptation au territoire de manière générale. Il peut être associé au fait que la race n'a pas fait l'objet d'une sélection pour des systèmes spécialisés et est restée adaptée à des systèmes plus économes. La dimension locale peut aussi renvoyer à un point de vue patrimonial, voire sentimental.

Dans le détail, il est possible de distinguer les principales motivations exprimées par les éleveurs, qu'ils combinent le plus souvent, chacun à sa manière (Tab. 1). Les deux premiers motifs renvoient aux aptitudes reconnues de la race : d'une part, la rusticité, les faibles besoins, l'autonomie et l'adaptation à des terres pauvres et, d'autre part, la qualité des produits, comme un lait riche à bonne qualité fromagère, la saveur de la viande, les carcasses de petit gabarit facilitant la découpe et la vente directe, etc. Le troisième motif renvoie à la transmission du patrimoine, à l'histoire locale et à la préservation de la biodiversité.

Les systèmes dans lesquels les éleveurs mobilisent la race sont très divers, de même que les effectifs des troupeaux. Une majorité a quelques vaches et cet élevage ne représente pas leur activité professionnelle principale. À l'inverse, d'autres sont essentiellement éleveurs et ont des troupeaux de taille variable, menés en systèmes laitiers ou allaitants. Ces différents systèmes correspondent néanmoins tous à une recherche de cohérence entre (i) usage de ressources locales, notamment ressources génétiques animales et ressources fourragères (ii) modalités de valorisation des produits de l'élevage (iii) durabilité de l'activité dans ses composantes environnementale, sociale et économique. Cela les amène à se distinguer des systèmes dit « conventionnels », à la fois par une économie en intrants et par leurs modalités de mise en marché originales.

Quel que soit le système d'élevage développé, les éleveurs professionnels de BPN ont mis en place des marchés spécifiques, en se constituant une clientèle, en développant des gammes de produits variés, empruntant pour cela des savoir-faire de transformation à d'autres territoires. La majorité des éleveurs laitiers vendent en circuits courts : sur les marchés, quelques-uns font de la vente directe, quelques-uns vendent en magasins bio ou auprès de restaurateurs. Dans tous les cas, les éleveurs ont dû se constituer une clientèle et la fidéliser, avec plus ou moins de facilité, selon la localisation géographique. La dimension locale «consommer Breton » peut d'ailleurs constituer un argument de vente pouvant être couplé avec l'argument « produit bio » dont la race locale peut renforcer l'image « naturelle ».

La vente de viande se fait aussi en direct, au sein de réseaux de clients constitués peu à peu. Pour cela, les éleveurs laitiers ont pu profiter du contact qu'ils ont avec leur clientèle en produits laitiers, sur les marchés en particulier. Pour les éleveurs en système strictement allaitant, les réseaux d'amis, la famille et le bouche à oreille sont le plus souvent sollicités.

\subsection{Des éleveurs laitiers du Grand Ouest remplacent leurs vaches Holstein par des vaches de race Montbéliarde ou Simmental Française}

Les races Montbéliardes et Simmental Française ne représentent que $2 \%$ des bovins du Grand Ouest mais, 
Tableau 1. Motivations des éleveurs en faveur de la race Bretonne Pie Noir.

Table 1. Breeders' reasons for choosing the Bretonne Pie Noir breed.

\begin{tabular}{lll}
\hline Catégories de motivation & Exemple de déclinaison des motivations par catégorie & $\begin{array}{l}\text { Nombre d'éleveurs pour lesquels } \\
\text { il y a occurrence de la catégorie }\end{array}$
\end{tabular}

Les aptitudes et le comportement des animaux Adaptation à son environnement ${ }^{* *}$, rusticité, facilité de conduite, autonomie de l'animal «s'en sort toute seule », produit peu, pertinente pour transformer ${ }^{* *}$ Qualité du lait, qualité pour faire des produits laitiers, meilleure valorisation de la viande, carcasse de petite taille, pertinente pour transformer ${ }^{* *}$

Patrimoine familial (un grand parent en avait par exemple), adaptation à son environnement ${ }^{* *}$, symbole d'une démarche, vache de la région, tradition, préférence pour la race locale, dimension esthétique il y a occurrence de la catégorie ${ }^{*}$

La qualité des produits issus de la race

Une vision de la conservation

\footnotetext{
${ }^{*}$ Il est important de noter le caractère indicatif de ce nombre, établi à partir d'une requête sur les éléments du matériau d'enquête codé comme se rapportant aux motivations pour choisir la race, qu'on retrouve chez 23 éleveurs et qui ne se veut pas représentatif de proportions rencontrées dans la population dans son ensemble.

** Certaines motivations nous semblent relever de plusieurs catégories et sont ainsi comptabilisées plusieurs fois.
}

Tableau 2. Répartition des motifs, cités en premier par les éleveurs, de la substitution de vaches de race Montbéliarde ou Simmental Française à des vaches de race Holstein au sein de 40 troupeaux laitiers du Grand Ouest de la France, selon le taux de substitution.

Table 2. Distribution of the first reasons expressed by 40 farmers in the 'Grand Ouest' area in France to replace their Holstein cows by Montbéliarde or French Simmental breeds, according to the substitution rate.

\begin{tabular}{|c|c|c|c|c|c|c|}
\hline Race de substitution & Part du cheptel substitué & \multicolumn{4}{|c|}{ Motif de la substitution } & Tota \\
\hline \multirow{3}{*}{ Montbéliarde } & $30-60 \%$ & 0 & 1 & 0 & 4 & 5 \\
\hline & $100 \%$ & 6 & 5 & 2 & 2 & 15 \\
\hline & Ensemble & 6 & 6 & 2 & 6 & 20 \\
\hline \multirow[t]{2}{*}{ Simmental Française } & $100 \%$ & 2 & 5 & 6 & 1 & 14 \\
\hline & Ensemble & 4 & 7 & 6 & 3 & 20 \\
\hline Ensemble des deux races & & 10 & 13 & 8 & 9 & 40 \\
\hline
\end{tabular}

aujourd'hui, environ $8 \%$ du cheptel de chacune de ces deux races est élevé dans ce grand bassin laitier, où elles étaient inexistantes jusque dans les années 1960 (Courdier et al., 2012).

Le Tableau 2 présente la répartition des motivations des éleveurs interviewés au sein de quatre catégories synthétiques définies à l'issue des entretiens. Elles résultent d'une analyse fondée sur trois variables : motivation principale du changement de race, durée de mise en œuvre du changement, évolution ou non du système de production. Nous avons choisi dans cet article de mettre l'accent sur les motivations des éleveurs.

La spécialisation laitière a eu pour conséquence une baisse des aptitudes fonctionnelles des vaches, et notamment leur fertilité (Le Mézec et Barbat, 2008). Cette dégradation, particulièrement marquée en race Holstein, a conduit un quart des éleveurs rencontrés à changer de race (Tab. 2). Ces éleveurs ont en effet atteint une situation de crise à laquelle ils n'ont vu d'autre issue que le choix d'une race perçue comme plus robuste, afin de « reprendre la main sur leur troupeau ». Face à cette situation, ils ont conduit une enquête au cours de laquelle ils ont cherché quelles ressources mobiliser. De précédents ajustements de conduite comme par exemple, le choix de taureaux Holstein susceptibles de transmettre des aptitudes de fertilité, des transformations de l'alimentation ou des actions en matière d'hygiène, ne leur avaient, en effet, pas permis d'améliorer la situation. Une grande majorité des éleveurs souligne la meilleure aptitude à la reproduction des races Montbéliarde et Simmental par rapport à la race Holstein, ce qui constitue pour eux une validation a posteriori de leur choix. Ces résultats issus d'entretiens sont corroborés par des analyses génétiques (Courdier et al., 2012) montrant notamment que les éleveurs de Montbéliardes du Grand Ouest ont porté une attention particulière à la fertilité dans le choix des taureaux d'insémination. En outre, pour la moitié des éleveurs de ce groupe, le changement de race s'est par la suite accompagné de 
l'adoption d'un système d'alimentation plus herbager. Dans un contexte d'accroissement des coûts de production et une situation d'incertitude quant au prix du lait (abandon des quotas laitiers), les éleveurs sont à la recherche (enquête) de moyens de diversifier et de sécuriser leurs revenus. Ainsi, dans nos entretiens, la recherche de mixité pour accroître le produit viande constitue le motif avancé le plus fréquemment pour le passage à la race Montbéliarde ou à la race Simmental (Tab. 2). Cette diversification, justifiée par la volonté de mieux utiliser les ressources fourragères, mobilisant l'une ou l'autre de ces deux races, correspond en fait à deux stratégies différentes. La moitié des éleveurs de ce groupe privilégient une utilisation intensive des ressources pour chacune des productions (lait et viande) et raisonnent la complémentarité des aptitudes non pas à l'échelle de chaque animal mais à l'échelle du troupeau, en maintenant une partie des vaches Holstein. L'autre moitié des éleveurs, en revanche, ont complètement éliminé les vaches Holstein de leur troupeau et ont misé sur des vaches aux aptitudes plus mixtes afin de valoriser le plus possible les surfaces fourragères.

Certains éleveurs, enfin, diminuent plus ou moins fortement le recours aux intrants, que leur enquête les ait conduits à limiter les coûts de production, à aller vers plus d'autonomie ou à réduire l'impact environnemental de leur activité d'élevage (ces trois considérations n'étant pas exclusives les unes des autres). Le remplacement de vaches Holstein par des vaches moins spécialisées peut s'inscrire dans cette volonté de « désintensification », ce motif étant cité à la troisième place par les éleveurs que nous avons interviewés (Tab. 2). Ce changement de race, souvent mûri sur une longue période, survient à la suite d'autres étapes de reconfiguration du système d'élevage et notamment du système fourrager, avec une baisse substantielle de la part de l'ensilage de maïs dans l'assolement et dans les rations, au profit de prairies temporaires (pour pâturage et ensilage) et de luzerne récoltée en foin. Dans ce choix d'une nouvelle race, les éleveurs interviewés mettent en avant la polyvalence et l'adaptation de l'animal au système qu'ils ont fait évoluer vers un recours accru à l'herbe.

Enfin le dernier groupe d'éleveurs rassemble ceux qui ont fait un " choix d'opportunité ». Ils ont découvert la race fortuitement, soit à la faveur de rencontres avec d'autres éleveurs ou de visites de salons professionnels et ont voulu «l'essayer » par l'acquisition de quelques vaches, soit à la faveur d'une reprise de troupeau dont ils ont choisi de garder les animaux. Bien que le choix de cette race ne soit pas anticipé, il peut toutefois donner lieu, pour ce dernier cas, à une évolution ultérieure de la conduite du troupeau.

\section{Discussion}

La mise en regard des deux cas étudiés visait à comprendre comment des éleveurs font le choix des ressources animales qu'ils mobilisent, notamment pour répondre à des enjeux de durabilité économique, sociale et environnementale, et à préciser le lien entre le caractère local des races et les modalités de cette réponse. Le cadre conceptuel mobilisé à cette fin nous invite à dépasser une entrée par les seules aptitudes des animaux pour considérer ces aptitudes en relation avec la situation, toujours émergente, des éleveurs. Cette situation, problématique au départ, entraîne en effet une enquête au cours de laquelle la race retenue in fine est identifiée comme une ressource. Elle entraîne de même des transactions, c'est-à-dire des interactions réciproques, avec l'environnement qui se transforme conjointement à la situation elle-même. C'est ainsi la notion même « d'animal localement adapté », plus propice à des systèmes d'élevage plus économes et autonomes, et généralement attribué aux animaux de races locales, que nous sommes conduits à réinterroger.

Les races locales sont en effet considérées comme localement adaptées comme si leur caractère local était un gage de cette adaptation. Un exemple emblématique en est fourni par le rôle que les races bovines laitières des Alpes du Nord ont tenu dans les années 1980 pour redynamiser à la fois l'élevage en zone de montagne, alors en déclin, et des filières fromagères alors en crise (Verrier, 1995). Nos résultats montrent que le caractère localement adapté n'est pas seulement le fait des aptitudes des races locales et des savoir-faire locaux qui leur seraient associés mais qu'il est aussi, et peut-être surtout, l'expression d'un processus actif d'adoption : pour être localement adaptée, il importe que la race soit localement adoptée, ce qui contribue également à la transformer, au fil des choix des éleveurs en matière de sélection des reproducteurs.

La race Bretonne Pie Noir a été la race dominante en Bretagne jusque dans les années 1950, avant d'être abandonnée en lien avec une spécialisation des activités agricoles et une forte intensification. Jugée insuffisamment productive, elle a peu à peu été considérée par la majorité des éleveurs et les organismes socio-professionnels comme non adaptée du fait du contexte de production en pleine transformation à cette époque. Les jeunes, en particulier, ont considéré que la BPN ne permettait pas de valoriser les prairies temporaires améliorées. Ils lui reprochaient son petit format et la difficulté à s'approvisionner en animaux ayant un potentiel laitier sûr, faute de contrôle laitier. Très vite, la race devint menacée (Quéméré, 2006). Les aptitudes qui faisaient d'elle une race très bien adaptée aux conditions de milieu difficiles d'avant-guerre ne présentaient plus autant d'intérêt dès lors que ces conditions ainsi que les attentes et les pratiques des éleveurs avaient changé. Inversement, depuis une vingtaine d'années, des éleveurs font le choix de remobiliser cette race dans des systèmes d'élevage très différents du système dominant actuel ainsi que du système traditionnel qui prévalait jusque dans les années 50. Pour ce faire, ils mobilisent des ressources extérieures à la Bretagne, telles que des savoir-faire fromagers, ou font le choix de conduire le troupeau dans un système allaitant. Au-delà d'une reconnaissance, dans le double sens de re-connaître et d'acquérir une certaine légitimité, des aptitudes de la BPN, il y a une ré-adoption de la race, localement, dans une Bretagne où les systèmes intensifs demeurent dominants. Les transactions engagées par ces éleveurs avec leur environnement leur permettent de développer des systèmes alternatifs viables qui passent également par la mise en place de modalités spécifiques de valorisation et de mise en marché des produits. La BPN est aussi adoptée au-delà de son berceau d'origine, puisque près d'un tiers des membres de l'UBPN sont exploitants agricoles dans d'autres départements (source : compte rendu de l'assemblée générale de l'UBPN 2014). Il serait intéressant de poursuivre le travail en prenant en compte ces éleveurs « hors berceau » et en comparant leurs modalités 
d'enquête ayant conduit au choix et à la mobilisation de la BPN avec celles des éleveurs bretons.

Pour faire face à une perte des aptitudes fonctionnelles des animaux de race Holstein, diversifier leurs productions, valoriser leurs ressources herbagères ou encore mettre en place un système plus respectueux de l'environnement, d'autres éleveurs du Grand Ouest ont identifié au cours de leur enquête des races originaires de l'Est comme ressources potentielles. Ce processus est essentiellement en lien avec la lecture de la presse spécialisée, leur expérience personnelle (stage antérieur dans l'Est) ou leur réflexion dans le cadre d'un groupe de développement. Il a ensuite été conforté par les coopératives d'insémination qui y ont vu des opportunités de diversification de leur activité. Les races Montbéliarde et Simmental française sont le plus souvent vues par les éleveurs comme leur permettant de mieux valoriser les ressources herbagères locales qui avaient été délaissées au profit de l'ensilage de maïs. L'herbe, qui n'était plus considérée comme une ressource, est de nouveau perçue et utilisée comme telle. Les éleveurs restent en revanche très intégrés dans les filières de commercialisation de grands groupes coopératifs qui se sont développés des années 1960-70 à nos jours. Ces deux aspects permettent de considérer que la Montbéliarde et la Simmental française sont localement adaptées au Grand Ouest dans un contexte d'élevage intensif. Au-delà, on peut dire que ces races ont été localement adoptées, comme en témoignent leur expansion démographique sur place et la mise en place de syndicats de race à l'initiative des éleveurs et sous la houlette des organismes de sélection concernés. Les concours organisés chaque année par ces syndicats favorisent les rencontres et l'émulation entre les éleveurs et, au-delà, constituent l'occasion d'une promotion locale de ces races. Ce sont aussi des lieux où les éleveurs du Grand Ouest participent à la gestion de la race et à son évolution.

D'autres cas de races localement adoptées auraient pu être mobilisés ici. Un des plus emblématiques est certainement celui de la race Simmental Française en Aubrac. En effet, elle compte, avec la race Aubrac, parmi les deux seules races autorisées par le cahier des charges du fromage AOP Laguiole et est très majoritaire en termes de production fromagère (moins de $5 \% \mathrm{du}$ Laguiole est fabriqué avec du lait de vaches Aubrac, LambertDerkimba et al., 2006). Ce choix de la race Simmental Française pour produire du Laguiole, à une époque où la race du cru évoluait radicalement vers des systèmes strictement allaitants, fut le résultat d'une enquête au sens où nous l'avons décrit plus haut (Vissac, 1993). Une différence importante est que, en Aubrac, l'enquête et le choix d'une race ont été opérés collectivement, alors que dans nos cas d'étude il s'agit de démarches individuelles. Ces différents cas illustrent bien la complexité de la notion d'adaptation locale et la diversité des facteurs qui entrent en jeu dans ces processus. On peut voir dans le développement d'une agriculture plus durable l'opportunité d'un maintien plus assuré, voire d'une relance, dans les années à venir, de races locales, à petits effectifs notamment, et ce d'autant plus qu'elles peuvent bénéficier d'aides publiques en faveur de la conservation de la biodiversité (comme les Mesures agro-environnementales en faveur de la protection des races menacées). Ce développement sera néanmoins peut-être autant le fait d'un projet issu d'une rencontre entre des hommes et une race dans des territoires variés, que de la mise en valeur des aptitudes d'animaux dans leur berceau d'origine. La notion de
« race localement adoptée » invite ainsi à promouvoir une lecture élargie d'un des principes de base de l'agro-écologie, qui encourage à la mobilisation des ressources locales, végétales comme animales (Altieri, 1995).

Remerciements. Nous remercions l'ensemble des personnes qui ont contribué à ces travaux, notamment, Marlène Courdier et Aurore Gérard, et tous les éleveurs rencontrés. Ces travaux ont bénéficié des financements du programme ANR O2LA et du programme COOPIGEN (Métaprogramme SELGEN INRA). Nous remercions également les relecteurs de notre article pour la pertinence de leurs remarques.

\section{Références}

Altieri MA. 1995. Agroecology: the science of sustainable agriculture, 2nd ed. Boulder, Colorado: Westview Press, p. 448.

Coquil X, Blouet A, Fiorelli JL, Bazard C, Trommenschlager JM. 2009. Conception de systèmes laitiers en agriculture biologique : une entrée agronomique. INRA Prod. Anim. 22(3): 221-234.

Courdier M, Moureaux S, Mugnier S, Gérard C, Gaillard C, Verrier E. 2012. L'extension des races bovines Montbéliarde et Simmental dans l'Ouest de la France : dynamique et conséquences génétiques. Rencontres Recherches Ruminants 19: 89.

Delaby L, Fiorelli JL. 2014. Élevages laitiers à bas intrants : entre traditions et innovations. INRA Prod. Anim. 27(2): 123-134.

Dewey J. 1938. Logic: the theory of inquiry. New York: Holt, Rinehart and Winston, p. 546.

Dumont B, Fortun-Lamothe L, Jouven M, Thomas M, Tichit M. 2013. Prospects from agroecology and industrial ecology for animal production in the 21 st century. Animal 7(6): 1028-1043. DOI: $10.1017 /$ S1751731112002418

Dumont B, Gonzales-Garcia E, Thomas M, Fortun-Lamothe L, Ducrot C, Dourmad JYet al. 2014. Forty research issues for the redesign of animal production systems in the 21st century. Animal 8(8): 1382-1393. DOI: $10.1017 / \mathrm{S} 1751731114001281$

Kaufmann JC. 1996. L'entretien compréhensif. Paris: Nathan, p. 128.

Labussière J, Richard PH. 1965. La traite mécanique. Aspects anatomiques, physiologiques et technologiques mise au point bibliographique. Ann. Zootech 14(1): 63-126.

Lambert-Derkimba A, Casabianca F, Verrier E. 2006. L'inscription du type génétique dans les règlements techniques des produits animaux sous AOC : conséquences pour les races animales. INRA Prod. Anim. 19(5): 357-370.

Le Mézec P, Barbat A. 2008 La fertilité des femelles laitières en France : regard sur 10 années et 37 millions d'IA. Journée de formation CSAGAD/IDELE, Paris 15 janvier 2008. Disponible sur http://www.agroparistech.fr/svs/genere/ger_genetique/csagad/ferti lite/fert 3 plm.pdf [consulté le 2 juin 2016].

Mattalia S, Barbat A, Danchin-Burge C, Brochard M, Le Mézec P, Minéry Set al. 2006. La variabilité génétique des principales races bovines laitières françaises : quelles évolutions, quelles comparaisons internationales ? Rencontres Recherches Ruminants 13: 239-246.

Piccand V, Cutullic E, Schori F, Kunz P, Thomet P. 2011. Quelle race pour produire et se reproduire en systèmes laitiers avec pâturage dominant et vêlages groupés ? Rencontres Recherches Ruminants 18: 409-412.

Quéméré P. 2006. La Bretonne Pie Noir: grandeur, décadence, renouveau. Paris: Éditions La France Agricole, p. 192.

Verrier E. 1995. La place des races bovines Abondance et Tarentaise dans une politique d'aménagement du territoire des Alpes du Nord. II - Une dynamique nouvelle en cours. Bulletin de l'Académie Vétérinaire de France 68: 193-200. 
Verrier E, Le Mezec P, Boichard D, Mattalia S. 2010. Évolution des objectifs et des méthodes de sélection des bovins laitiers. Bulletin de l'Académie Vétérinaire de France 163(1): 73-78. Disponible sur http://www.academie-veterinaire-defrance.org.
Vissac B. 1993. Société, race animale et territoire entre les théories et 1'Histoire : réflexions sur une crise. Nat. Sci. Soc. 1(4): 282-297.

Zask J. 2008. Situation ou contexte ? Une lecture de Dewey. Revue Internationale de Philosophie 3: 313-328.

Citation de l'article : Couix N, Gaillard C, Lauvie A, Mugnier S, Verrier É. 2016. Des races localement adaptées et adoptées, une condition de la durabilité des activités d'élevage. Cah. Agric. 25: 650009. 